\title{
Two Types of High Inductive Electromagnetic Stimulation and Their Different Effects on Endothelial Cells
}

\author{
J. PRŮCHA ${ }^{1,2}$, J. SKOPALIK ${ }^{3,4}$, V. SOCHA ${ }^{5}$, L. HANÁKOVÁ ${ }^{1,5}$, L. KNOPFOVÁ ${ }^{6}$, \\ K. HÁNA ${ }^{1}$
}

${ }^{1}$ Department of Information and Communication Technologies in Medicine, Faculty of Biomedical Engineering, Czech Technical University in Prague, Prague, Czech Republic, ${ }^{2}$ Department of Health Care Disciplines and Population Protection, Faculty of Biomedical Engineering, Czech Technical University in Prague, Prague, Czech Republic, ${ }^{3}$ Department of Human Pharmacology and Toxicology, Faculty of Pharmacy, University of Veterinary and Pharmaceutical Sciences Brno, Czech Republic, ${ }^{4}$ Department of Biomedical Engineering, Faculty of Electrical Engineering and Communication, Brno University of Technology, Brno, Czech Republic, ${ }^{5}$ Laboratory of Human Factors and Automation in Aviation, Faculty of Transportation Sciences, Czech Technical University in Prague, Prague, Czech Republic, ${ }^{6}$ Department of Experimental Biology, Faculty of Science, Masaryk University, Brno, Czech Republic

Received August 9, 2018

Accepted April 15, 2019

Epub Ahead of Print June 6, 2019

\section{Summary}

Effects of low-frequency electromagnetic fields (LF EMF) on the activation of different tissue recovery processes have not yet been fully understood. The detailed quantification of LF EMF effects on the angiogenesis were analysed in our experiments by using cultured human and mouse endothelial cells. Two types of fields were used in the tests as follows: the LF EMF with rectangular pulses, 340-microsecond mode at a frequency of $72 \mathrm{~Hz}$ and peak intensity $4 \mathrm{mT}$, and the LF EMF with sinusoidal alternating waveform $5000 \mathrm{~Hz}$, amplitude-modulated by means of a special interference spectrum mode set to a frequency linear sweep from 1 to $100 \mathrm{~Hz}$ for $6 \mathrm{~s}$ and from $100 \mathrm{~Hz}$ to $1 \mathrm{~Hz}$ return also for $6 \mathrm{~s}$, swing period of 12 second. Basic parameters of cultured cells measured after the LF EMF stimulus were viability and proliferation acceleration. Both types of endothelial cells (mouse and human ones) displayed significant changes in the proliferation after the application of the LF EMF under conditions of a rectangular pulse mode. Based on the results, another test of the stimulation on a more complex endothelial-fibroblast coculture model will be the future step of the investigation.

\section{Key words}

Low frequency-electromagnetic fields • Induced electric currents - Neoangiogenesis • Endothelial cells

\section{Corresponding author}

L. Hanáková, Department of Information and Communication Technologies in Medicine, Faculty of Biomedical Engineering, Czech Technical University in Prague, nám. Sítná 3105, 27201 Kladno, Czech Republic. Email: hanakle1@fbmi.cvut.cz

\section{Introduction}

Time-varying magnetic field, produced by electric field through the mediation of electromagnetic induction, causes induced electric currents in conductive media. The use of this concept in various medical applications is currently encountered in commonly used therapeutic tools. One of the first therapeutic uses of induced coupled electric field and current stimulation was employed in the treatment of patients suffering from pseudoarthrosis and non-unions (Basset et al. 1977). This therapeutic approach is currently applied to the physical therapy and rehabilitation (Iannitti et al. 2013), wound healing (Cheing et al. 2014), pain management (Strauch et al. 2009, Andrade et al. 2016), and many other areas (Shupak et al. 2003).

In addition, the pulsed electromagnetic fields 
(PEMF) are also used for these purposes. The nature of therapeutic devices taking advantage of the PEMF is generally based on using rapid and accurately defined time change in the magnetic field causing bounces of induced electrical voltage and consequently induced electric currents in the tissues treated (for more information see work by Shupak et al. 2003 and Oschman 2016). It has been suggested that the PEMFinduced intracellular effects originate at the cell membrane, since membrane constituents should be much better detectors of electric fields than isolated molecules in solutions. The induced electric field and corresponding currents in the extracellular medium could alter ion binding to macromolecules situated in the membrane, influence the ligand-receptor association and modify the general membrane transport processes (Tenforde 1996). However, these phenomena can propagate through subsequent ion channel opening and long-time affecting of mitochondrial metabolism or mitotic activity of nuclear components (Prucha et al. 2018).

In addition to the PEMF mentioned, the concept of sinusoidal electromagnetic fields is also employed, indicating comparable effects in the recovery of tissues in respective studies. These studies are mostly aimed at the bone regenerative medicine and demonstrate effects such as improved viability, proliferation and osteogenic differentiation of mesenchymal stromal cells (Liu et al. 2013), increased metabolism with inhibited resorption of both metaphyseal and diaphyseal bone tissue (Zhou et al. 2014), and reduction in the patient healing time (Ledda et al. 2015).

Special case of sinusoidal electromagnetic fields (SEMF) and induced electric currents exerting the sinusoidal course, called interference currents, principally represent an amplitude modulation of the harmonic current obtained based on the interference of currents from two sources of harmonic, i.e. sinusoidal induced electric currents exerting a small difference in their frequencies. Since the difference can vary in time, the frequency of the amplitude modulation will also vary. In our case, we can affect the cellular model investigated by the basic (carrier) sinusoidal frequency of $5 \mathrm{kHz}$ as well as by lower frequencies (ELF - extra low frequencies) linearly varying from $1 \mathrm{~Hz}$ to $100 \mathrm{~Hz}$ and back from $100 \mathrm{~Hz}$ to $1 \mathrm{~Hz}$, always for time intervals of $6 \mathrm{~s}$, i.e. with a $12 \mathrm{~s}$ period, provided that ELF have the sinusoidal course. In a number of studies, this is referred to as interference therapy, which can have analgesic (Defrin et al. 2005) and bone healing effects (Ganne 1988).
Even though the above mentioned electric fieldbased phenomena are known to drive the development and regeneration of many tissues, especially of the cartilage, bone or dermal tissue (Levin 2003, Nuccitelli 1988), there is still a lack of quantitative analyses of EMF interactions with certain cell types, despite positive results of PEMF, SEMF and other types of EMF from various biological and clinical studies in the last 40 years. In this connection, it is important to consider that endothelial cells are principal structural elements in the process of vessel building, and that proliferation of endothelial cells is critical for all regenerative actions (Banno and Yoder 2014) such as the secondary angiogenesis after ischaemia and scar formation. Unfortunately, the electromagnetic stimulation of tissue related to endothelial cells is still relatively poorly described biophysical phenomenon. In addition, the choice of important EMF parameters for the effective stimulation of a specific cell type, such as endothelial cells, is also missing.

Based on the facts mentioned, the presented work was focused on two types of EMFs and their effects on endothelial cells. In comparison with chondrocytes or osteocytes, the endothelial cells and their interaction with specific EMFs have not yet been sufficiently described (Levin 2003, Nuccitelli 1988). The situation could be explained by comparatively difficult in vitro culturing of endothelial cells and rather high cell prices. However, the presented article is primarily focused on the "macrocellular" level of the cell interaction with EMFs, i.e. the characteristics detected are the viability and acceleration of the proliferation. The choice of monitored parameters is based on standard approaches, where the viability is the first criterion in all the tests. The in vitro proliferation acceleration is a key parameter, which can correlate with improved angiogenesis in real tissues (Johnson and Wilgus 2017, Tahergirabi and Khazaei 2012). The topics considered here have not yet been studied and could have a positive impact on understanding of the effects of discussed physical therapy methods.

\section{Materials and Methods}

\section{Samples preparation}

For the presented study, two types of endothelial cells were obtained, namely human umbilical vein endothelial cells (HUVEC) lines and mouse microvascular lines (MS1) manufactured by Lonza Inc., NJ, USA. The cells were precultivated in $25 \mathrm{~cm}^{2}$ culture 
flasks (Invitrogen - Thermo Fisher Scientific Inc., MA, USA). The basal medium for endothelial cells CC-3162 and set of growth factors CC-4176 were prepared and mixed in accordance with manufacturer's instructions (Lonza Inc., NJ, USA). Prior to experiments with electromagnetic applicators, all cell types were cultivated for at least 8 weeks. All cell cultures were passaged by trypsinization after reaching $90 \%$ confluency.

Cultivation of cell samples and application of electromagnetic stimuli in vitro

Endothelial MS1 and HUVEC cells were inoculated onto standard 96-well tissue culture plates (TPP Techno Plastic Products AG, Switzerland). As initial density, 2500 cells $/ \mathrm{cm}^{2}$ (800 cells/well) was chosen. Samples were subsequently cultivated in a CO2CELL 190 cultivation incubator (BMT Medical Technology Ltd., Czech Republic) at $37{ }^{\circ} \mathrm{C}$ and an air atmosphere with $5 \%$ of carbon dioxide. The same cultivation medium as in case of sample preparation was used.

In this study, three plates were used for each of the two cell types, i.e. MS1 and HUVEC. At the beginning of each experiment with the cells, $3.8 \times 10^{6}$ cells were inoculated into 16 wells on three separate plates. The cells on the first plate were used as controls (CTR cell group), the cells on the second plate were exposed to pulsed electromagnetic field (EM1 cell group), and those on the third plate were exposed to the specific sinusoidal electromagnetic field with interference effects (EM2 cell group).

The EM1 cell group was exposed to effects of the EMF characterized by generating pulsed induced electric currents. In this case, the peak of the magnetic field was of $4 \mathrm{mT}$, pulse width of $340 \mu \mathrm{s}$, with rectangular shape of the exciting pulse. A series of pulses was generated at a frequency of $72 \mathrm{~Hz}$ for $2.5 \mathrm{~s}$ (the so-called burst). The burst was followed by a short pause of $0.5 \mathrm{~s}$ and this cycle was periodically repeated. In this setting, induced electric current pulses had a value of $4.5 \mathrm{mV} / \mathrm{cm}$ in the peak and in cells they produced current density of about $0.27 \mathrm{~A} / \mathrm{m}^{2}$ (for assumed cell specific conductivity of $0.6 \mathrm{mS}$ ). Parameters of these clinically verified pulsed currents were based on works by Basset et al. (1987) and Basset et al. (1974).

The EM2 cell group was exposed to the action of EMF characterized by generating sinusoidal induced electric currents with amplitude modulation provided by means of the interference. In this case, the magnetic field peak was of $6 \mathrm{mT}$. The harmonic carrier frequency of $5000 \mathrm{~Hz}$ was modulated by frequencies of 1 to $100 \mathrm{~Hz}$ with $100 \%$ premodulation. For a period of 6 s, a linear frequency increase (sweep) was produced from $1 \mathrm{~Hz}$ to $100 \mathrm{~Hz}$; for a period of next $6 \mathrm{~s}$ a linear drop from $100 \mathrm{~Hz}$ to $1 \mathrm{~Hz}$ followed. The induced interference currents (peak value in one polarity) of the electric field intensity of $18.8 \mathrm{mV} / \mathrm{cm}$ produced a current density of $1.1 \mathrm{~A} / \mathrm{m}^{2}$ (for the cell specific conductivity of $0.6 \mathrm{mS}$ ).

The two mentioned electromagnetic fields applied were low-frequency, high inductive electromagnetic fields according to described characteristics. For the generation of the electromagnetic fields mentioned above, the VAS-07 instrument (Embitron, Ltd, Czech Republic) with an appropriate coil applicator was used, see Figure 1. All the parameters in the application were measured with the help of Maschek ESM100 (TMV SS Ltd, Czech Republic) and Gaussmeter GM05 (Hirst Magnetic Instruments Ltd., Great Britain) instruments. In both groups, application time of the electromagnetic stimulation was of $2 \times 90 \mathrm{~min}$ daily for both HUVEC and MS1. During application of pulsed or sinusoidal EMF, applicators (Fig. 1) were integrated into the $\mathrm{CO}_{2}$ CELL 190 incubator. Exposure of cells samples to EMF was carried under standard in vitro conditions $\left(37{ }^{\circ} \mathrm{C}, 5 \% \mathrm{CO}_{2}\right.$ in air atmosphere, $\mathrm{PCO}_{2}=40 \mathrm{mmHg}$ (5.3 $\mathrm{kPa}), 95 \%$ humidity).

In total, there were four experiments executed under identical conditions for each type of cells (HUVEC and MS1) exposed to specific magnetic field as well as for control group. For each type of cells, independent analyses were carried out on days 2, 4, 6 and 8 of respective experiment. For purposes of the analyses on specific day, 4 wells of each plate were used. For outline of the employed types of in vitro cultures, variants of irradiation and number of particular in vitro wells see Fig. 1. The detailed method of evaluating the cells and dynamics of their growth is shown below.

\section{Evaluation of endothelial cell viability and morphology stability}

The first measurement, which was a prerequisite for detailed viability examinations, was based on a temperature test. Liquid medium on the bottom of the culture chamber near the stimulating coil was measured to assure safe temperature during application of the electromagnetic stimulation. High-precision GMH 3710 Pt100 Thermometer (GHM Messtechnik GmbH Standort Greisinger, Germany) was used for precise temperature 


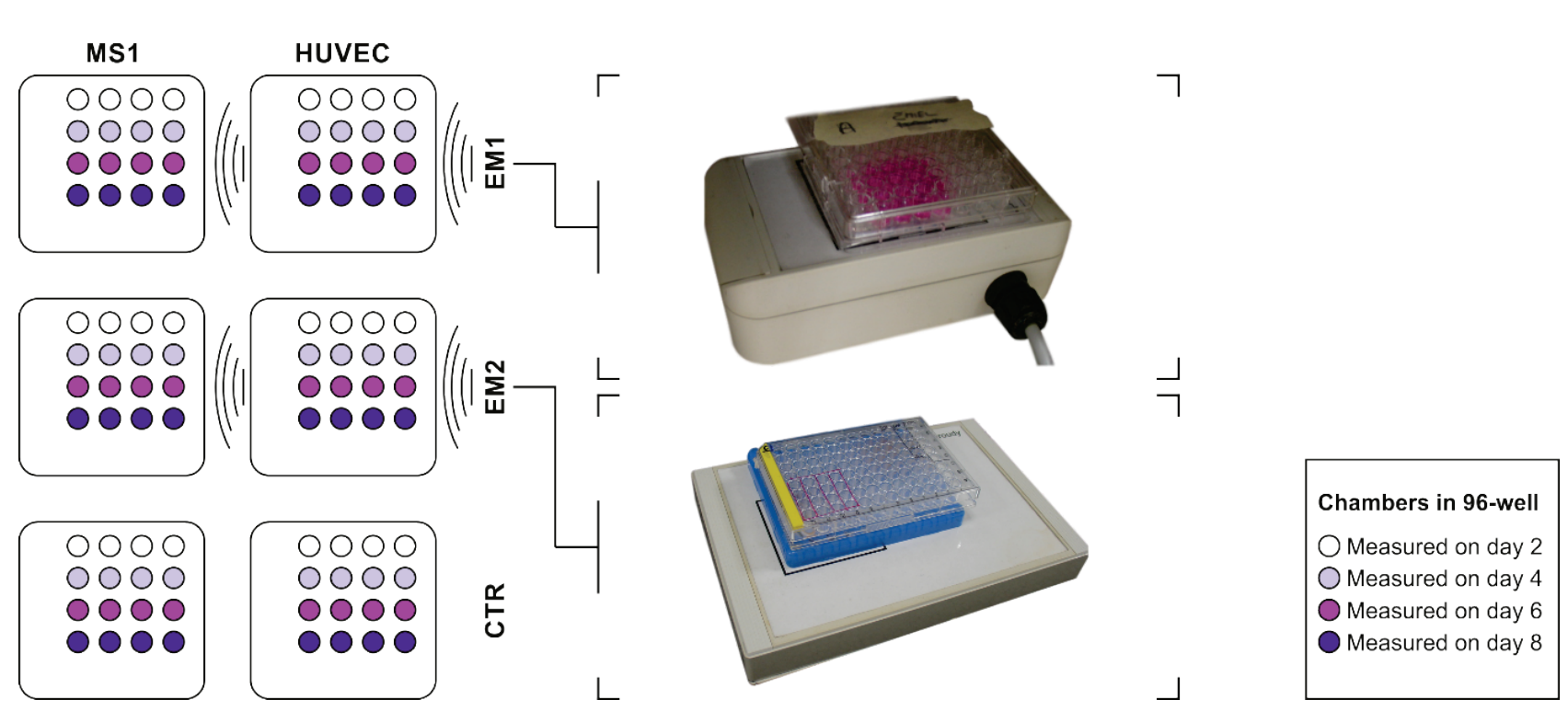

Fig. 1. Schematic depiction of the experiment with an outline of in vitro culture types and electromagnetic field applicators. HUVEC: human umbilical vein endothelial cells; MS1: mouse endothelial cells; CTR: control group; EM1: cell group exposed to pulsed electromagnetic field (4 mT magnetic field peak, $340 \mu \mathrm{s}$ pulse width, $72 \mathrm{~Hz}$ frequency and $1 \mathrm{~A} / \mathrm{m}^{2}$ amplitude of the current density); EM2: cell group exposed to sinusoidal electromagnetic field $(6 \mathrm{mT}$ magnetic field peak, harmonic carrier frequency of $5000 \mathrm{~Hz}$ modulated by frequencies of 1 to $100 \mathrm{~Hz}$ with $100 \%$ premodulation, $6 \mathrm{~s}$ period of linear frequency increase/drop and $1.1 \mathrm{~A} / \mathrm{m}^{2}$ amplitude of the current density).
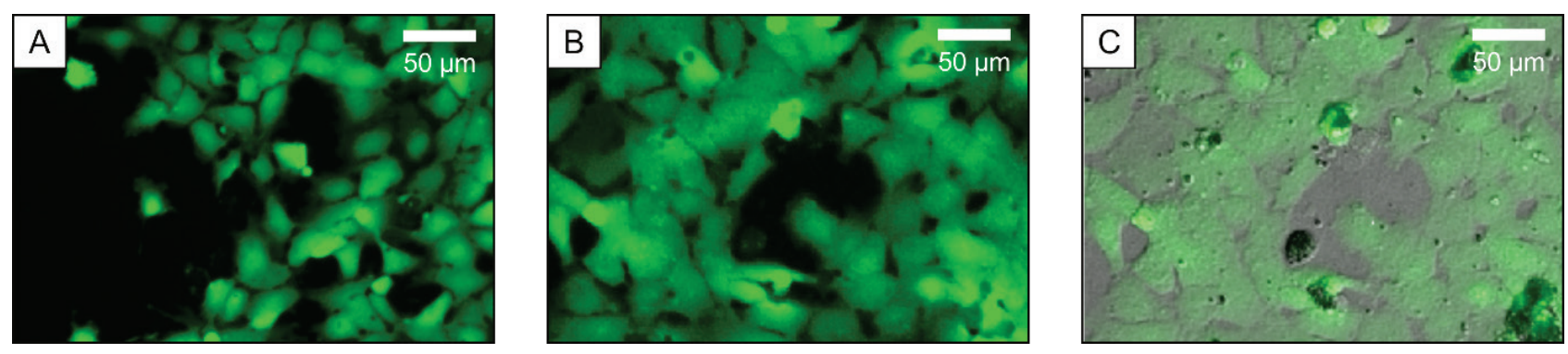

Fig. 2. Illustrative scans of growing human vain endothelial cells control group under fluorescence mode (A), cells stimulated by pulsed electromagnetic field under fluorescence mode (B) and under phase contrast mode (C).

measurements in small volumes. The cell viability was quantified with the use of the LIVE/DEAD cell viability kit (Thermo Fisher Scientific Inc., MA, USA). The cells were stained according to manufacturer's instructions.

Additional analysis of expression of HUVEC cell surface ligands (cluster of differentiation CD31, CD144, CD34) on EMF stimulated and control cells was conducted by flow-cytometer Cytomics FC500 (Beckman-Coulter, Inc., IN, USA). Cell surface CD were stained by monoclonal antibody against $\mathrm{CD} 31$ conjugated to phycoerythrin (1:100 dilutions), antibody against CD144 conjugated to allophycocyanin (1:100 dilutions) and antibody against CD34 conjugated to Fluorescein isothiocyanate (1:100 dilutions). All commercial antibodies were obtained from Miltenyi Biotec, Germany. Unstained cells and cells incubated with isotype-matched controls were used as reference between fluorescent positive and fluorescent negative populations. Cells size and granularity of EMF stimulated and control cells were compared from basic dot-plot (forward versus side scatter). At least 2000 cells in the non-debris gate were selected for evaluation of $\mathrm{CD}$ markers expression in each cells sample, values of fluorescence intensity per one cell were converted to $\mathrm{ABC}$ standard fluorescence unit using QuickC software (Bangs Laboratories, Inc., IN, USA) and final datasets were presented by histograms (Fig. 3). For more details of cytometric methods see work by Klabusay et al. (2007).

\section{Endothelial cell proliferation}

The endothelial cell proliferation was evaluated by using two approaches: the indirect quantification of the cell growth by using the metabolic activity measurement and the direct cell counting measurement 
by using a calcein method.

The first method was the cellular metabolic activity measurement in a given cultivation well. The measurement was implemented with the use of commercial tetrazolium dye colorimetric assay (MTT) for cellular growth and viability (Sigma-Aldrich, Inc., MO, USA). This was a standard test of the metabolic activity based on a 4-hours incubation of cells with MTT (Sylvester 2011). The well was rinsed with dimethyl sulfoxide in accordance with the manufacturer's instruction. The final measurement of the absorption (at $530 \mathrm{~nm}$ ) for each single cultivation well was done by means of the Elisa-reader Paradigm instrument (Arnold O. Beckman, Inc., CA, USA). As a result, the relative deviation of the metabolic activity from the control sample (the MTT index) was obtained. Therefore, the data for a further statistical analysis were MTT indices recorded on the given day separately for each well within the group examined.

The second method measured the number of live cells by counting under a fluorescence microscope after staining with the Calcein Acetoxymethyl ester dye (Sigma-Aldrich, Inc., MO, USA). The data collection principle was based on the determination of the area covered with cells by using fluorescence microscopy images. For this purpose, Arsenal AIF1T fluorescence microscope (Arsenal - Microscopy Ltd., Czech Republic) with corresponding software tool was used. Within the framework of measurements carried out on particular days, the surface area covered with cells was evaluated as a mean value from 10 fields of view for each well. In this way, the data for further statistical evaluation presented the mean occupation of the surface area with cells (expressed as $\mu \mathrm{m} 2$ per well surface area) and obtained in measurement on each day for each well separately in the group studied. Results of fluorescence microscopy records for stimulated and non-stimulated cells are exemplified in Figure 2.

\section{Statistical analysis}

For purposes of the presented study, the statistical analysis was aimed at a comparison of differences between the groups of interest, i.e. EM1, EM2 and CTR. To this end, the one-way analysis of variance (ANOVA) was employed to examine differences between group means. The ANOVA was particularly conducted to explore the impact of the high inductive magnetic stimulation on levels of the cell metabolic activity (by using the MTT method) and on the cell surface for three groups observed on particular days. To check the homogeneity of the variance, the Bartlett test was used, verifying the null hypothesis that the observed data originate from normal distribution with the same variance against the alternative hypothesis that not all the data have the same variance. The Bartlett test shows that there is no strong evidence against the assumption of the equal variance between groups at a $5 \%$ significance level.

Error-plot was used for the visualization of the course of measured values, depicting the mean value and respective deviation in the measurement characterized by standard error. To represent the dynamics of the growth, the distribution of particular measurements at particular times was fit to the linear course in the group followed.

For statistical analysis and associated calculations, the Matlab environment (MATLAB R2017a, MathWorks, Inc., MA, USA) and its statistical toolbox was used.

\section{Results}

\section{Endothelial cell viability and stability of morphology}

Temperatures over $37^{\circ} \mathrm{C}$ could change the cell metabolism or induce the cell death in the long term. Setting of the final version of the applicator in the $\mathrm{CO}_{2}$ incubator and final setting of the distance between the surface of the applicator and the chamber bottom (Fig. 1) helped us to achieve effective EMF application with no over-heating of the liquid in the chamber. The long-term temperature curve was strictly between 36.7 and $37.1^{\circ} \mathrm{C}$, which was comparable with control chambers without EMF.

The viability of the human and mouse endothelial cells after the 3-day EMF application was quantified as $98.3 \pm 1.1 \%$ and $98.8 \pm 0.5 \%$ respectively (versus $98.1 \pm 1.3 \%$ and $98.85 \pm 1.1 \%$ in control cells).

The stability of typical endothelial surface markers (CD31, CD34, CD144) after the pulsed and sinusoidal EMF application was evaluated by the quantitative flow-cytometer (histograms from one cell sample in Figure 3 show typical examples of the CD positivity and negativity on control and irradiated cells). All the samples of endothelial cells before and after the EMF procedure display almost identical profiles of $C D$ markers. Results from flow quantitative cytometry also indicated that morphology of cells remains the same.

\section{MTT index-based endothelial cell proliferation}

In the case of the evaluation of differences 

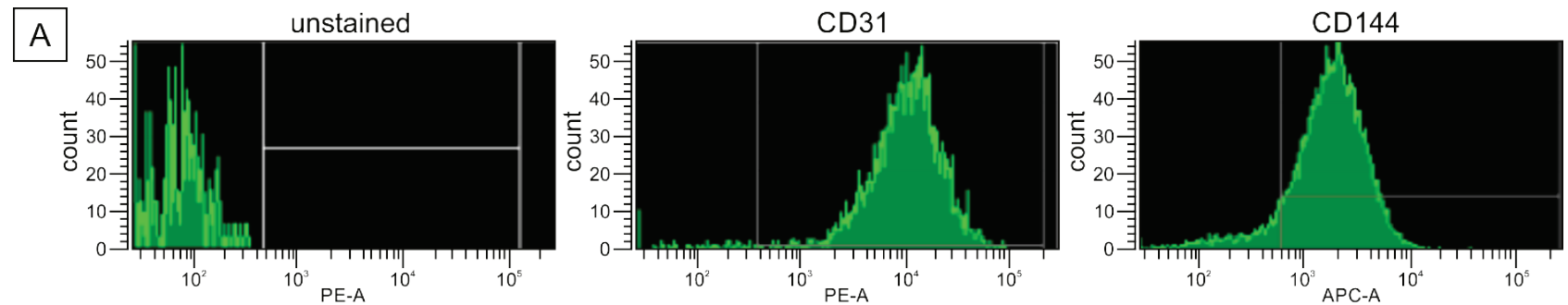

unstained
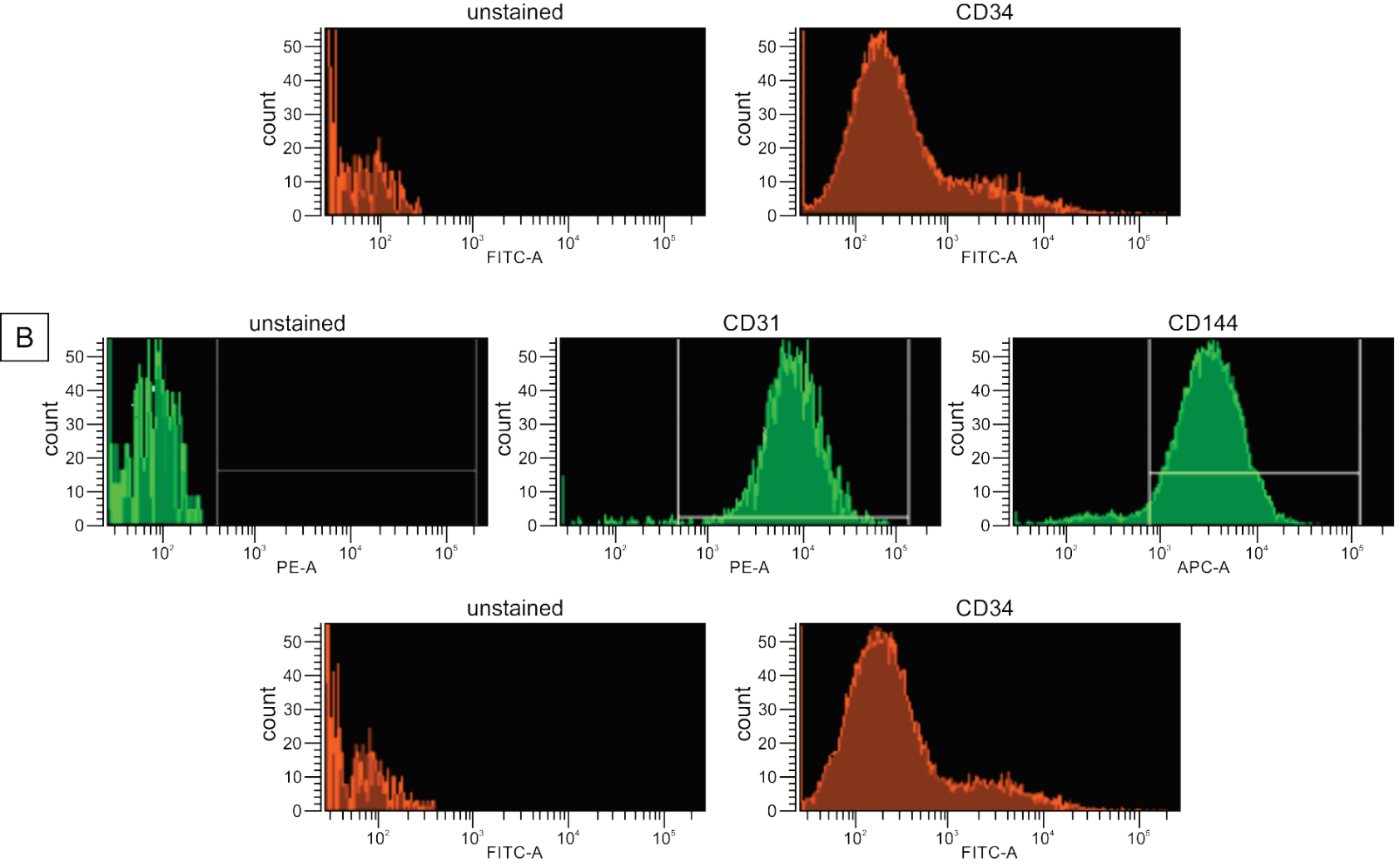

Fig. 3. Illustrative histograms characterising stability of specific clusters of differentiation markers (CD31, CD144 and CD34) on human endothelial control cells (A) and human endothelial cells exposed to pulsed electromagnetic field (B). PE-A: phycoerythrin; APC-A: allophycocyanine; FITC-A: fluorescein isothiocyanate.

between groups in human endothelial cells (Fig. 4A), the difference in mean MTT values was compared by using one-way ANOVA test between the groups studied (EM1, EM2 and (TR) at particular time points, i.e. on days 4, 6 and 8 . The values of the MTT index observed on the second day exerted no variability in measurements, which limits to a certain extent the use of ANOVA test or other statistical tests employed for evaluation of the difference between groups.

From the results shown in Figure 4 it follows that in case of EM1 there was different MTT index course within the monitored interval compared to EM2 and CTR. The results suggest single trend. The cell number, estimated by MTT test, increased under pulsed EMF stimulation (EM1 cell group), while under sinusoidal EMF stimulation (EM2 cell group) it remained similar as in control unstimulated cells. At the same time, it is possible to state that on the second day, there were no significant differences between the groups. Detailed statistical results and significant differences from individual days and for individual cell types are described in the following text.

When evaluating differences between groups in human endothelial cells (Fig. 4A) based on the measurement on the second day, it is possible to conclude that between groups EM1 (mean=0.048, $\mathrm{SD}=0.002$ ), EM2 (mean=0.05, $\mathrm{SD}=0$ ) and CTR (mean=0.05, $\mathrm{SD}=0$ ) there is no statistically significant difference.

There was a statistically significant difference in the MTT index between the three observed groups on day $4, \mathrm{~F}_{(2,9)}=10.39, \mathrm{p}=0.004$. The effect size calculated by using squared eta was $\eta^{2}=0.69$. This means that approximately $69 \%$ of the variance was caused by an independent variable. Post-hoc comparisons by using 
the Tukey HSD test indicated that the mean score for EM1 (mean=0.213, $\mathrm{SD}=0.021$ ) was significantly different from EM2 (mean=0.127, $\mathrm{SD}=0.015)$ and $\mathrm{CTR}$ (mean $=0.135, \mathrm{SD}=0.022$ ) at a significance level $\mathrm{p}<0.05$, since the confidence interval for the difference between the means of EM1 and EM2 was $\mathrm{CI}_{1-2}(0.0251,0.1309)$ and between EM1 and CTR was $\mathrm{CI}_{1-\mathrm{CTR}}(0.0183,0.1242)$. The post-hoc analysis also showed that EM2 and CTR did not differ significantly from each other, $\mathrm{CI}_{\text {2-CTR }}(-0.0597,0.0462)$.

In the case of measurements on day 6, statistically significant difference in the MTT index between the three groups was observed, $F_{(2,9)}=9.22$, $\mathrm{p}=0.006$. The effect size calculated by using squared eta was $\eta^{2}=0.67$. Post-hoc comparisons indicated that the mean score for EM1 (mean=0. 365, $\mathrm{SD}=0.037$ ) was significantly different from EM2 (mean $=0.288$, $\mathrm{SD}=0.038$ ) and CTR (mean=0.302, $\mathrm{SD}=0.045$ ) at a significance level $\mathrm{p}<0.05$, since the confidence interval for the difference between the means of EM1 and EM2 was $\mathrm{CI}_{1-2}(0.0184,0.1366)$ and between EM1 and CTR was $\mathrm{CI}_{1 \text {-CTR }}(0.0209,0.1391)$. The post-hoc analysis also demonstrated that EM2 and CTR did not differ significantly from each other, $\mathrm{CI}_{2-\mathrm{CTR}}(-0.0566,0.0616)$.
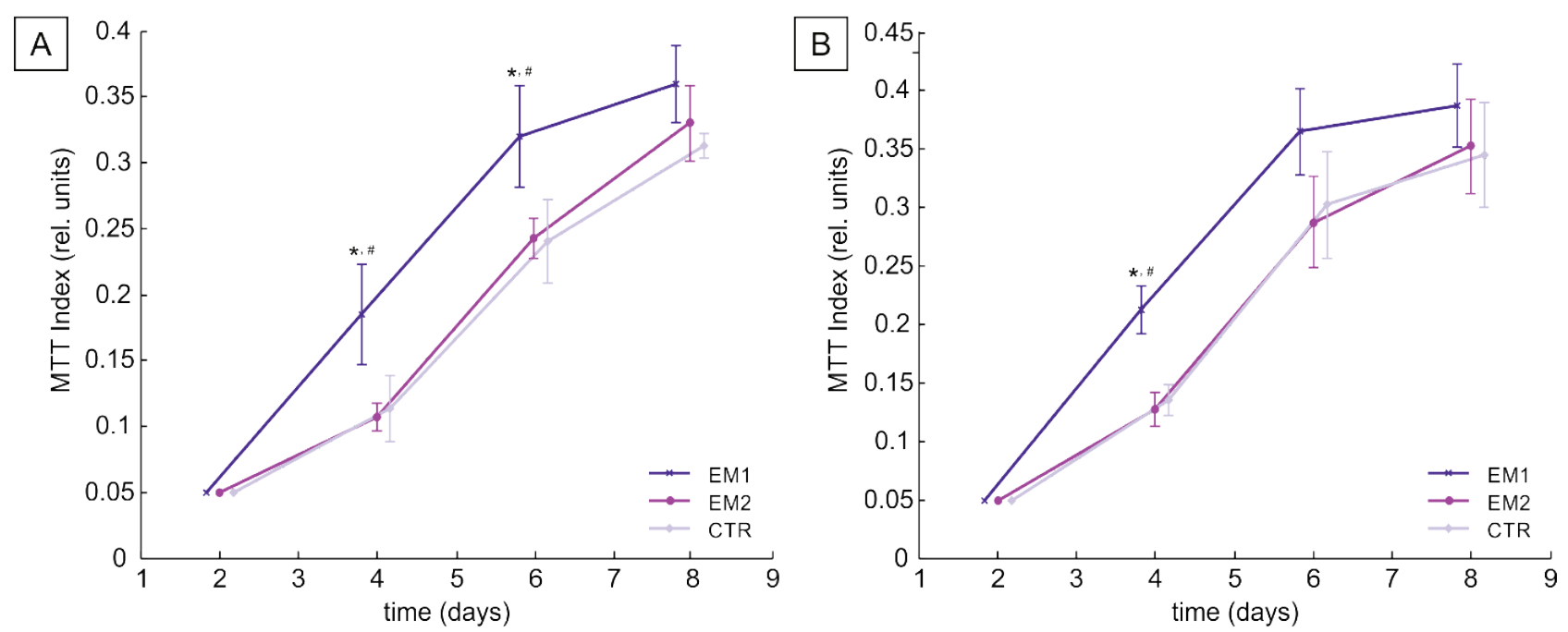

Fig. 4. Distribution of metabolic activity (MTT Index) of cells measured in particular days for control group (CTR), cell group exposed to pulsed electromagnetic field (EM1) and cell group exposed to sinusoidal electromagnetic field (EM2) of human (A) and mouse (B) endothelial cells. Values are expressed as means \pm SD. ${ }^{*} p<0.05$ vs. CTR group; ${ }^{*} p<0.05$ vs. EM2 group.
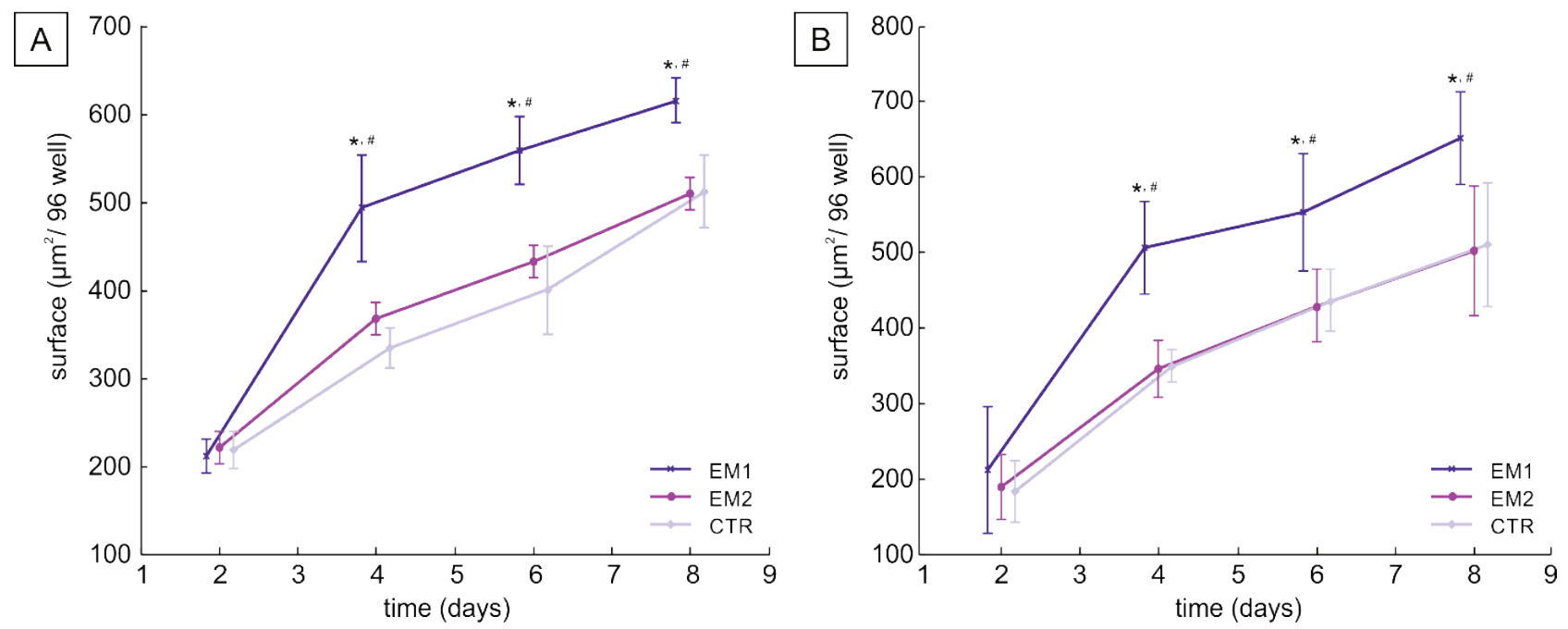

Fig. 5. Distribution of surface area covered with cells measured in particular days for control group (CTR), cell group exposed to pulsed electromagnetic field (EM1) and cell group exposed to sinusoidal electromagnetic field (EM2) of human (A) and mouse (B) endothelial cells. Values are expressed as means \pm SD. ${ }^{*} p<0.05$ vs. CTR group; ${ }^{*} p<0.05$ vs. EM2 group. 
In the last measurement on day 8 , no statistically significant difference was observed in the MTT index between the groups studied, $\mathrm{F}_{(2,9)}=3.93, \mathrm{p}=0.06$. The effect size calculated by using squared eta was $\eta^{2}=0.46$.

In the case of the evaluation of differences between groups in mouse endothelial cells (see Fig. 4B), one-way ANOVA test was used to compare the difference in mean values of the MTT index in the groups examined (EM1, EM2 and CTR) at particular time points, i.e. on days 4,6 and 8. The values of the MTT index observed on the second day exerted, similarly as in the case of the human endothelial cells, no variability in measurements. In the case of the measurement on the second day, it is thus possible to state that between groups EM1 (mean $=0.048, \quad \mathrm{SD}=0.002), \quad \mathrm{EM} 2$ (mean $=0.05, \mathrm{SD}=0$ ) and $\mathrm{CTR}$ (mean $=0.05, \mathrm{SD}=0$ ) there is no statistically significant difference and the values of these measurements are identical with those for human endothelial cells.

In case of measurements on day 4 , statistically significant difference in the MTT index between the three groups was observed, $\mathrm{F}_{(2,9)}=32.54, \mathrm{p}<0.001$. The effect size calculated by using squared eta was $\eta^{2}=0.88$. Posthoc comparisons indicated that the mean score for EM1 (mean $=0.212, \mathrm{SD}=0.021$ ) was significantly different from EM2 (mean $=0.127, \mathrm{SD}=0.015$ ) and CTR (mean $=0.135$, $\mathrm{SD}=0.013$ ) at a significance level $\mathrm{p}<0.05$, since the confidence interval for the difference between the means of EM1 and $\mathrm{EM} 2$ was $\mathrm{CI}_{1-2}(0.052,0.1176)$ and that between EM1 and CTR was $\mathrm{CI}_{1 \text {-CTR }}(0.0449,0.1101)$. The post-hoc analysis also showed that EM2 and CTR did not differ significantly from each other: $\mathrm{CI}_{2-\mathrm{CTR}}(-0.0401$, 0.0251).

In the measurements performed on days 6 and 8 , no statistically significant difference was observed in MTT indices between the groups studied. In these cases, one-way ANOVA statistics for the evaluation of differences between groups resulted in $\mathrm{F}_{(2,9)}=4.09$, $\mathrm{p}=0.06$ in case of day 6 and $\mathrm{F}_{(2,9)}=1.26, \mathrm{p}=0.328$ in case of day 8 .

\section{Surface-based endothelial cell proliferation}

Similarly, as in the preceding cases, in the case of the evaluation of differences between groups in human endothelial cells (Fig. 5A), one-way ANOVA test was used to compare differences between mean values of the surface area covered with cells for particular groups (EM1, EM2 and CTR) at particular time points, i.e. on the days 4,6 and 8 .
Similarly, as concerning the MTT index, single trend for both human and mouse endothelial cells is observable. In case of pulsed EMF stimulation (EM1 cell group), there was an increase of the monitored parameter during the measured days. This group statistically differed from cells exposed to sinusoidal EMF (EM2 cell group) and CTR. At the same time, it is possible to state that EM2 and CTR exhibited similar trend during the monitored time period and individual measured days showed no significant difference between mean values of the surface area covered with cells for these two groups. Further, it is apparent that there was no significant difference between all monitored groups on the second day. Detailed results including respective statistics are included in the following text.

When evaluating differences between groups in human endothelial cells, for day 2 there were no statistically significant differences between EM1 (mean=211, SD=19.37), EM2 (mean=221.5, SD=18.2) and CTR (mean=218, $\mathrm{SD}=21.19$ ) found by evaluation of surfaces covered with cells, $\mathrm{F}_{(2,9)}=0.29, \mathrm{p}=0.75$. The effect size calculated by using squared eta was also very low: $\eta^{2}=0.061$.

In case of measurements on day 4 , statistically significant difference in the surface covered with cells was observed for the three groups: $F_{(2,9)}=23.75, p<0.001$. The effect size calculated by using squared eta was $\eta^{2}=0.84$. Post-hoc comparisons with the use of the Tukey HSD test indicated that the mean score for EM1 (mean=485.5, $\mathrm{SD}=36.37$ ) was significantly different from EM2 (mean=374.75, $\mathrm{SD}=35.52$ ) and CTR (mean=334.25, $\mathrm{SD}=22.6)$ at a significance level $\mathrm{p}<0.05$, since the confidence interval for the difference between the means of EM1 and EM2 was $\mathrm{CI}_{1-2}(47.31,174.18)$ and between EM1 and CTR it was $\mathrm{CI}_{1-\mathrm{CTR}}(87.81,214.68)$. The posthoc analysis also showed that EM2 and CTR did not differ significantly from each other: $\mathrm{CI}_{2-\mathrm{CTR}}(-22.93$, 103.93).

Similar results were also observed in measurements carried out on day 6. One-way ANOVA showed that there was statistically significant difference in the surface covered with cells between the groups studied: $\mathrm{F}_{(2,9)}=20.51, \mathrm{p}<0.001$. The effect size calculated by using squared eta was $\eta^{2}=0.82$. Post-hoc comparisons indicated that the mean score for EM1 (mean=558.5, $\mathrm{SD}=38.30$ ) was significantly different from $\mathrm{EM} 2$ (mean=431.75, SD=31.97) and CTR (mean=407.5, $\mathrm{SD}=36.82$ ) at a significance level $\mathrm{p}<0.05$, since the confidence interval for the difference between the means 
of EM1 and EM2 was $\mathrm{CI}_{1-2}(56.06,197.43)$ and that between EM1 and CTR was $\mathrm{CI}_{1-\mathrm{CTR}}(80.31,221.68)$. The post-hoc analysis also showed that EM2 and CTR did not differ significantly from each other: $\mathrm{CI}_{2-\mathrm{CTR}}(-46.43$, 94.93).

Statistical difference in surfaces covered with cells between groups measured on day 8 was also observed, $\mathrm{F}_{(2,9)}=32.32, \mathrm{p}<0.001$. The effect size calculated by using squared eta was $\eta^{2}=0.88$. Post-hoc comparisons indicated that the mean score for EM1 (mean=615.5, $\mathrm{SD}=25.21)$ was significantly different from those for EM2 (mean=499.5, $\mathrm{SD}=21.42$ ) and CTR (mean=501.75, $\mathrm{SD}=23.21$ ) at a significance level $\mathrm{p}<0.05$, since the confidence interval for the difference between the means of EM1 and EM2 was $\mathrm{CI}_{1-2}(69.93,162.07)$ and that between EM1 and CTR was $\mathrm{CI}_{1-\mathrm{CTR}}(67.68,159.82)$. The post-hoc analysis also showed that EM2 and CTR did not differ significantly from each other: $\mathrm{CI}_{2-\mathrm{CTR}}(-48.32$, 43.82).

Following the evaluation of the surface covered with cells regarding mouse endothelial cells (Fig. 5B), the analysis by one-way ANOVA test demonstrated no statistically significant differences between EM1 (mean=186.75, SD=39.42), EM2 (mean=189, SD=43.62) and CTR (mean=182.75, $\mathrm{SD}=41.51)$ on day 2: $\mathrm{F}_{(2,9)}=0.023, \mathrm{p}<0.97$.

In case of measurement carried out on day 4 , statistically significant difference in the surface covered with cells was observed for the three groups: $F_{(2,9)}=25.61$, $\mathrm{p}<0.001$. The effect size calculated by using squared eta was $\eta^{2}=0.85$. Post-hoc comparisons indicated that the mean scores for EM1 (mean=506.25, $\mathrm{SD}=45.24$ ) were significantly different from EM2 (mean=346.25, $\mathrm{SD}=37.72$ ) and CTR (mean=349.25, $\mathrm{SD}=21.31$ ) at a significance level $\mathrm{p}<0.05$, since the confidence interval for the difference between the means of EM1 and EM2 was $\mathrm{CI}_{1-2}(88.59,231.4)$ and that between EM1 and CTR was $\mathrm{CI}_{1-\mathrm{CTR}}(85.59,228.40)$. The post-hoc analysis also showed that EM2 and CTR did not differ significantly from each other: $\mathrm{CI}_{2-\mathrm{CTR}}(-74.4,68.4)$.

On day 6 , statistically significant difference in the surface covered with cells between groups studied was also observed: $F_{(2,9)}=9.32, p=0.006$. The effect size calculated by using squared eta was $\eta^{2}=0.67$. Post-hoc comparisons indicated that the mean scores for EM1 (mean=553.75, SD=47.62) were significantly different from EM2 $($ mean=429.5, $\quad \mathrm{SD}=47.45)$ and $\mathrm{CTR}$ (mean=437, $\mathrm{SD}=41.54$ ) at a significance level $\mathrm{p}<0.05$, since the confidence interval for the difference between the means of EM1 and EM2 was $\mathrm{CI}_{1-2}(34.16,214.33)$ and that between EM1 and CTR was $\mathrm{CI}_{1-\mathrm{CTR}}(26.66,206.83)$. The post-hoc analysis also showed that EM2 and CTR did not differ significantly from each other: $\mathrm{CI}_{2-\mathrm{CTR}}(-97.58,82.58)$.

Concerning the last measurements carried on day 8 , statistically significant difference was also observed, $\mathrm{F}_{(2,9)}=8.763, \mathrm{p}=0.007$. The effect size calculated with the use of squared eta was $\eta^{2}=0.66$. Posthoc comparisons showed that the mean score for EM1 (mean=651.75, SD=60.53) was significantly different from EM2 (mean=489.25, $\mathrm{SD}=63.09)$ and $\mathrm{CTR}$ (mean=498.25, $\mathrm{SD}=61.45$ ) at a significance level $\mathrm{p}<0.05$, since the confidence interval for the difference between the means of EM1 and EM2 was $\mathrm{CI}_{1-2}(40.681,284.31)$ and that between EM1 and CTR was $\mathrm{CI}_{1-\mathrm{CTR}}(31.68$, 275.31). The post-hoc analysis also showed that EM2 and CTR did not differ significantly from each other: $\mathrm{CI}_{2-\mathrm{CTR}}(-130.81,112.81)$.

\section{Discussion}

In general, the presented results demonstrate significant PEMF effects on the proliferation concerning both mouse and human endothelial cells. The EM1 cell group demonstrated statistically significant differences against EM2 and CTR groups during the period of interest. Although in terms of the MTT index these differences were not observed in the case of HUVEC on day 8 and MS1 on days 6 and 8 , it is possible to conclude that, in general, EM1 exerts effects on the proliferation. This fact was also supported by the evaluation with counting under the fluorescence microscope.

In contrast to this, the study of samples after the sinusoidal EMF application (EM2 group), demonstrated no statistically significant difference in the proliferation of cells against CTR. Statistically significant differences from CTR were absent even in the case of MTT index or when following the surface area occupied by cells throughout the entire period of observations.

In general, our experimental results show that LF PEMF does not affect the cell viability of endothelial cells. The field is biocompatible and usable for several day-to-day applications without visible senescence or degradation of endothelial cells.

The acceleration of endothelial cell growth is not characteristic for all the types of EMF stimuli. For example, the application of DC electric fields based on direct setting of cathode and anode into the culture 
medium in vitro $(150-400 \mathrm{mV} / \mathrm{mm})$ caused reorientation and elongation of endothelial cells, but no enhanced proliferation (Bai et al. 2004, Xiong et al. 2015). An intense picosecond pulsed electric field (pulse duration of $800 \mathrm{ps}, 3 \mathrm{~Hz}$ recurrence rate and field strength of 2000-4000 kV/mm) had negative effects on the migration as well as proliferation of HUVEC and suspended the cell cycle in the G2/M phase (Wu et al. 2016). On the other hand, PEMF with $4.5 \mathrm{~ms}$ pulses repeated at $15 \mathrm{~Hz}$ (magnetic flux density rising from 0 to $12 \mathrm{G}$ ) activated the production of FGF-2 (well-described angiogenic mediator) after longer periods of time; the authors also described measurable in vitro effects of accelerated HUVEC proliferation and promising angiogenic in vivo effects (Tepper et al. 2004).

In the presented study, both types of endothelial cells (mouse and human) demonstrated significant changes in the growth activity of the cells in in vitro chambers after the PEMF stimulation, i.e. concerning the EM1 cell group. On the other hand, only minor changes in the cell growth were found after the sinusoidal EMF stimulation. EMF was effective with $340-\mu$ s pulses repeated at $72 \mathrm{~Hz}$ (magnetic density rising from 0 to $4 \mathrm{mT}$ ). These parameters are not far from those used by Tepper et al. (2004), and the results of the effective proliferation activation are also similar.

The details of PEMF key effects, which play their role in the activation of the cell proliferation, have not yet been clearly explained. In this study, we focused on recognition and exact quantification of basic cellular phenomena (cells surviving, proliferation, stability and cells area decrease), not yet on investigation of internal molecular mechanisms which are on background of these cellular phenomena. A number of theoretical models have been proposed, attempting to explain the interaction mechanisms between PEMF and molecular complex in internal compartments and membranes of HUVEC cell bodies or other cells.

Some key PEMF-induced intracellular effects can originate at the cell membrane, since membrane constituents should be much better detectors of applied electric fields than isolated molecules in the solution. The induced electric field and corresponding currents in the extracellular medium could alter ion binding to macromolecules situated in the membrane, influence the ligand-receptor association and modify the general membrane transport processes (Tenforde 1996). We started with measurement of membrane depolarization and changes of membrane permeability on cells after
PEMF stimulation. However, complex statistical data for accepting our hypothesis and characterising the permeability for specific size of molecules are not included in the presented paper and still need to be produced by more complex experiments and larger statistical evaluation. We have several indications that these membrane phenomena can propagate by means of modifying ion channel opening and long-term affecting of mitochondrial metabolism or mitotic activity of nuclear components (Prucha et al. 2018).

Our study has three important limitations. In the first place, we used only one type of endothelial cells from each human or mouse organisms. In the second place, we prepared the endothelial cell culture based on traditional $2 \mathrm{D}$ in vitro culture material; more sophisticated experiments were recently published based on the use of $3 \mathrm{D}$ tubes, and more appropriately approximating the real geometry of the vasculature (Zargar et al. 2016). In the third place, in our basic in vitro culture, we did not use any complex mix of all cell types, which are near to in vivo capillaries and veins (Kaessmeyer et al. 2017). It is very likely that the inductive electromagnetic stimulation (about $50 \mathrm{~Hz}$ ) should have double activating in vivo effects: effect on endothelial cells and an indirect effect based on the activation of keratinocytes (their activity and paracrine extracts can promote building of complex endothelial and sub-endothelial structures similar to real capillaries). These facts may be subjected to further research in the field of interest discussed here.

Under conditions of current densities at least about $1 \mathrm{~A} / \mathrm{m}^{2}$ (high-inductive electromagnetic field), the electromagnetic field inducing the coupled electric pulsed field and electric pulsed current exert demonstrable clinical effects anyway, see also results obtained by Prucha et al. (2018). Common clinical data report for example effects supporting the capillary return (Pitr and Prucha 2001). Recent studies were carried out with cell cultures of F11 type peripheral sensory neurons (Prucha et al. 2018). These were hybridoma cells, embryonal ganglia of the rat spine cord posterior roots representing a model of a specific subgroup of sensory neurons responsible for the transfer of stimuli of different modalities, including stimuli associated with the nociception and pain resulting from an inflammation injury, degenerative disease of the locomotor apparatus but also healing, recovery and regeneration etc. It was demonstrated that in a stimulation of inflammation, with the use of bradykinin as an external noxious agent and 
with the presence of the pulsed electromagnetic field of the type corresponding to pulsed EMF used in the present work, the amplitude of the increase in the concentration of calcium inside of the cell is reduced and the time period of the enhanced calcium concentration is extended, Consequently, the cell reacts to the inflammatory noxious agent by a finer, more cautious and also more physiological reaction with decreased risks of the destruction. In the presented study of cultures of endothelial cells, we found that the induced electric currents or currents of the single pulse type according to Bassett et al. (1987) and Bassett et al. (1974) support the growth of both human and mouse endothelial cells and their metabolic activity. In content of the presented study, the inducted currents with pulse width of $340 \mu \mathrm{s}$, frequency $72 \mathrm{~Hz}$ and about $1 \mathrm{~A} / \mathrm{m}^{2}$ amplitude of the current density represent physical intervention comparable by its parameters with distance electrotherapy (Prucha et al. 2004, Pitr and Prucha 2001). The demonstrated result in the presented paper could be of one mechanisms of clinical effects leading to supporting the capillary return and healing in the application of the inductive electromagnetic stimulation.

\section{Conflict of Interest}

The authors claim no conflict of interest.

\section{Acknowledgements}

This study was supported by the grant No. 16-28784A "Influence of high inductive magnetic stimulation on symptoms of locomotive apparatus degenerative diseases" and grant No. FV20422 "Development of nanofibrous scaffolds providing the application of cellular products, including physical stimulation effect, for treatment of the chronic wounds" co-financed by Czech Ministry of Industry and Trade.

\section{References}

ANDRADE R, DUARTE H, PEREIRA R, LOPES I, PEREIRA H, ROCHA R, ESPREGUEIRA-MENDES J: Pulsed electromagnetic field therapy effectiveness in low back pain: A systematic review of randomized controlled trials. Porto Biomedical Journal 1: 156-163, 2016.

BAI H, MCCAIG CD, FORRESTER JV, ZHAO M: DC electric fields induce distinct preangiogenic responses in microvascular and macrovascular cells. Arterioscler Thromb Vasc Biol 24: 1234-1239, 2004.

BANNO K, YODER MC: Tissue regeneration using endothelial colony-forming cells: promising cells for vascular repair. Pediatr Res 3: 647-661, 2014.

BASSETT CAL, PAWLUK RJ, PILLA AA: Acceleration of fracture repair by electromagnetic fields: A surgically noninvasive method. Ann N Y Acad Sci 238: 242-262, 1974.

BASSETT CAL, PILLA AA, PAWLUK RJ: A non-operative salvage of surgically-resistant pseudarthroses and non-unions by pulsing electromagnetic fields. Clin Orthop Relat Res 124: 128-143, 1977.

BASSETT CAL: Low energy pulsing electromagnetic fields modify biomedical processes. Bio Essays 6: 36-42, 1987.

CHEING GL-Y, LI X, HUANG L, KWAN RLC, CHEUNG KK: Pulsed electromagnetic fields (PEMF) promote early wound healing and myofibroblast proliferation in diabetic rats. Bioelectromagnetics 35: 161-169, 2014.

DEFRIN R, ARIEL E, PERETZ C: Segmental noxious versus innocuous electrical stimulation for chronic pain relief and the effect of fading sensation during treatment. Pain 115: 152-160, 2005.

GANNE JM: Stimulation of bone healing with interferential therapy. Aust J Physiother 34: 9-20, 1988.

IANNITTI T, FISTETTO G, ESPOSITO A, ROTTIGNI V, PALMIERI B: Pulsed electromagnetic field therapy for management of osteoarthritis-related pain, stiffness and physical function: clinical experience in the elderly. Clin Interv Aging 8: 1289-1293, 2013.

JOHNSON KE, WILGUS TA: Vascular endothelial growth factor and angiogenesis in the regulation of cutaneous wound repair. Adv Wound Care (New Rochelle) 83: 283-290, 2017.

KAESSMEYER S, SEHL J, KHIAO-IN M, MERLE R, RICHARDSON K, PLENDL J: Subcellular interactions during vascular morphogenesis in 3D cocultures between endothelial cells and fibroblasts. Int J Mol Sci 18: E2590, 2017.

KLABUSAY M, SUKOVA V, COUPEK P, BRYCHTOVA Y, MAYER J: Different levels of CD52 antigen expression evaluated by quantitative fluorescence cytometry are detected on B-lymphocytes, CD 34+ cells and tumor cells of patients with chronic B-cell lymphoproliferative diseases. Cytometry B Clin Cytom 72: 363-370, 2007. 
LEDDA M, D’EMILIA E, GIULIANI L, MARCHESE R, FOLETTI A, GRIMALDI S, LISI A: Nonpulsed sinusoidal electromagnetic fields as a noninvasive strategy in bone repair: The effect on human mesenchymal stem cell osteogenic differentiation. Tissue Engineering Part C: Methods 21: 207-217, 2015.

LEVIN M: Bioelectromagnetics in morphogenesis. Bioelectromagnetics 24: 295-315, 2003.

LIU C, YU J, YANG Y, TANG X, ZHAO D, ZHAO W, WU H: Effect of $1 \mathrm{mT}$ sinusoidal electromagnetic fields on proliferation and osteogenic differentiation of rat bone marrow mesenchymal stromal cells. Bioelectromagnetics 34: 453-464, 2013.

NUCCITELLI R: Ionic currents in morphogenesis. Experientia 44: 657-666, 1988.

OSCHMAN JL: Electricity and magnetism in diagnosis and therapeutics. In Energy Medicine: The Scientific Basis. Elsevier: Churchill Livingstone, London, 2016, pp 75-99.

PITR K, PRUCHA J: Regression of pain of the locomotor apparatus and other effects associated with application of distance electrotheraphy. (In Czech) Rehabil Fyz Lek 8: 70-85, 2001.

PRUCHA J, HAVEL V, PITR K: The physical conditions of the distant electrotherapy application in patients with metallic endoprosthesis. (In Czech) Rehabil Fyz Lek 11: 184-188, 2004.

PRUCHA J, KRUSEK J, DITTERT I, SINICA V, KADKOVA A, VLACHOVA V: Acute exposure to high-induction electromagnetic field affects activity of model peripheral sensory neurons. J Cell Mol Med 22: 1355-1362, 2018.

SHUPAK NM, PRATO FS, THOMAS AW: Therapeutic uses of pulsed magnetic-field exposure: a review. URSI Radio Science Bulletin 76: 9-32, 2003.

STRAUCH B, HERMAN C, DABB R, IGNARRO LJ, PILLA AA: Evidence-based use of pulsed electromagnetic field therapy in clinical plastic surgery. Aesthet Surg J 29: 135-143, 2009.

SYLVESTER PW: Optimization of the tetrazolium dye (MTT) colorimetric assay for cellular growth and viability. Methods Mol Biol 716: 157-68, 2011.

TAHERGORABI Z, KHAZAEI M: A review on angiogenesis and its assays. Iran J Basic Med Sci 15: 1110-1126, 2012.

TENFORDE TS: Interaction of elf magnetic fields with living systems. In: Handbook of Biological Effects of Electromagnetic Fields. C POLK, E POSTOW (Eds.), CRC Press, New York, 1996, pp 189-234.

TEPPER OM, CALLAGHAN MJ, CHANG EI, GALIANO RD, BHATT KA, BAHARESTANI S, GAN J, SIMON B, HOPPER RA, LEVINE JP, GURTNER GC: Electromagnetic fields increase in vitro and in vivo angiogenesis through endothelial release of FGF-2. FASEB J 18: 1231-1233, 2004.

WU Y, HUA Y, XIONG Z, WU L, YAO C, ZHANG R, WANG Z: Picosecond pulsed electric field inhibits the proliferation and migration, induces $\mathrm{G} 2 / \mathrm{M}$ phase arrest and promotes apoptosis in human umbilical venous endothelial cells. (In Chinese) Xi Bao Yu Fen Zi Mian Yi Xue Za Zhi (Chinese Journal of cellular and molecular immunology) 32: 319-322, 2016.

XIONG GM, DO AT, WANG JK, YEOH CL, YEO KS, CHOONG C: Development of a miniaturized stimulation device for electrical stimulation of cells. J Biol Eng 9: 1-14, 2015.

ZARGAR R, NOURMOHAMMADI J, AMOABEDINY G: Preparation, characterization, and silanization of 3D microporous PDMS structure with properly sized pores for endothelial cell culture. Biotechnol Appl Biochem 63: 190-199, 2016.

ZHOU J, MA X-N, GAO Y, YAN J-L, SHI W-G, XIAN CJ, CHEN K-M: Sinusoidal electromagnetic fields promote bone formation and inhibit bone resorption in rat femoral tissues in vitro. Electromagn Biol Med 35: 75-83, 2014. 